\title{
ON THE EXISTENCE OF WEAK CUT POINTS IN PLANE CONTINUA
}

\author{
F. BURTON JONES ${ }^{1}$
}

Suppose that $K$ is the sum of two circles in the plane tangent at the point $x$. (They may be "internally" or "externally" tangent.) Let $D$ be the complementary domain of $K$ having $K$ as its boundary; let $Q$ be one of the other complementary domains; and let $M$ be the complement of $D+Q$. If $p$ is a point of $M$ not in $\bar{Q}$ and $q$ is a point of $Q$, it is clear that $x$ cuts $p$ from $q$ in $M+Q$. In fact, since $M$ is locally connected, the existence of such a cut point follows from a wellknown separating point theorem in plane topology. ${ }^{2}$ However, if, instead of being a locally connected continuum, $K$ is merely a continuum (possibly indecomposable), then $M$ is not necessarily locally connected and the existence of such a cut point is not so evident or its ambiguous location gives no clue to the proof of its existence. The purpose of this paper is to state and prove a cut point existence theorem of this general nature. As is frequently the case with separating point theorems, when one weakens the hypothesis by discarding the local connectedness requirement, one must weaken the conclusion by replacing the notion of "separating point" by the notion of "cut point." But since the two notions are equivalent in the presence of local connectedness, one usually gets a stronger theorem, and this is the case here.

Definitions and notation. Let space be a 2 -sphere and let $S$ denote the set of all points of the space. ${ }^{3}$ If $a$ and $b$ are points of a continuum $M$, the subset $X$ of $M-(a+b)$ is said to cut a from $b$ in $M$ provided that every subcontinuum of $M$ containing $a$ and $b$ contains a point of $X$. When $X$ contains only one point, it is called a $c u t$ point. If $M$ is a continuum, by a complementary domain of $M$ is meant a component of $S-M$. If $D$ is a connected open set and $Q$ is a connected subset of $S-\bar{D}$, by the outer boundary of $D$ with respect to $Q$ is meant the boundary of the complementary domain of $\bar{D}$ containing $Q$.

Presented to the Society, April 26, 1958; received by the editors February 21, 1958.

1 An NSF Senior Postdoctoral Fellow.

2 E.g., Theorem 43 , p. 222, of [1].

${ }^{3}$ For compact continua, the theorem and proof are applicable to other spaces such as the plane, any space satisfying Axioms $0-5$ of [1], etc. 
Theorem. ${ }^{4}$ Suppose that $D$ and $Q$ are different complementary domains of a continuum $M$ such that the boundary $B$ of $Q$ is the outer boundary of $D$ with respect to $Q$. Then if $p \in M-B$ and $q \in Q$, there exists a point $x$ of $B$ which cuts $p$ from $q$ in $M+Q$.

Proof. ${ }^{5}$ Let $\rho$ denote a distance function for $S$. If there exists a simple sequence $\alpha$ of closed subsets of $M+Q$ each of which cuts $p$ from $q$ in $M+Q$ such that $\alpha$ converges to a point $x$ of $B$, then $x$ cuts $p$ from $q$ in $M+Q$. For otherwise if some subcontinuum $H$ of $M+Q$ contains $p+q$ but not $x$, there exists a term $A$ of $\alpha$ such that $\rho(A, H)$ is positive and $A \cdot I I \neq 0$. This is a contradiction. So if the theorem is false no such sequence $\alpha$ exists.

Now assume that the theorem is false. Hence there exists a positive number $\epsilon$ such that if $L$ is a closed subset of $M+Q$ whose diameter is $3 \epsilon$ or less and whose distance from $B$ is less than $\epsilon$ then $L$ does not cut $p$ from $g$ in $M+Q$. This follows from the above paragraph and the compactness of $M+Q$. For convenience it is assumed that $\epsilon$ is less than $\rho(p+q, B)$ and also less than the diameter of $B$.

There exists a simple closed curve $J$ lying in $D$ such that one of its two complementary domains (call this domain $E$ ) contains $M$ and for each point $z$ of $B, \rho(z, J)<\epsilon / 2$. There exists a finite collection $G$ of circular regions covering $B$ such that the center of each element of $G$ belongs to $B$ and each element of $G$ has diameter $\epsilon$. Let $C_{1}$, $C_{2}, \cdots, C_{n}$ denote the boundaries of the elements of $G$. Since $B$ is a continuum, some subset of $C_{1}+C_{2}+\cdots+C_{n}$ is a simple closed curve in $E$ separating $B$ from $q$; hence $\left(C_{1}+C_{2}+\cdots+C_{n}\right) \cdot(E+J)$ separates $p$ from $q$ in $E+J$. So there exists a finite collection $T_{1}, T_{2}, \cdots, T_{m}$ of arcs lying in $E+J$ such that (1) for each $i, T_{i}$ intersects $J$ in only its end points, (2) for each $i$, there exists a $j$ such that $T_{i} \subset C_{j}$ and (3) $\sum T_{i}$ separates $p$ from $q$ in $E+J$.

Now $T_{1}$ does not separate $p$ from $q$ in $E+J$ for if it $\operatorname{did} T_{1} \cdot(M+Q)$ would cut $p$ from $q$ in $M+Q$, would be of diameter $3 \epsilon$ or less, and would lie at a distance of less than $\epsilon$ from $B$ contrary to the definition of $\epsilon$. There exists a simple domain $E_{1}$ whose boundary $J_{1}$ is a subset of $J+T_{1}$ such that $E_{1} \subset E-E \cdot T_{1}$ and $p+q \subset E_{1}$. Proceeding by induction, suppose that $E_{k}$ is a simple domain whose boundary $J_{k}$ is a subset of $J+T_{1}+\cdots+T_{k}$ such that $E_{k} \subset E_{k-1}-E_{k-1} \cdot \sum_{i=1}^{k} T_{i}$ and $p+q \subset E_{k}$ but such that $T_{k+1} \cdot \bar{E}_{k}$ does separate $p$ from $q$ in $\bar{E}_{k}$. (This

${ }^{4}$ One aspect of how close this theorem comes to being false is illustrated in Fig. 24, p. 223, of [1].

s The reader will find Theorems 11-15, pp. 163-167 and Theorems 28-34, pp. $197-203$, of [1] helpful in verifying some of the steps in this argument. 
must happen for some $k$ less than $m$, since $\sum T_{i}$ separates $p$ from $q$ in $E+J$.) It follows ${ }^{6}$ that some component $T_{k+1}^{\prime}$ of $T_{k+1} \cdot \bar{E}_{k}$ separates $p$ from $q$ in $\bar{E}_{k}$. This set $T_{k+1}^{\prime}$ is an arc with its end points $a$ and $b$ in $J_{k}$. Now $a$ either belongs to $J$ or, for some natural number $i \leqq k$, belongs to $T_{i}$ (which has its end points in $J$ ); likewise $b$ either belongs to $J$ or, for some natural number $j \leqq k$, belongs to $T_{j}$ (which has its end points in $J$ ). Let $p q$ be an arc in $E_{k}$ from $p$ to $q$. Since $p q$ intersects $T_{k+1}^{\prime}$, let $p^{\prime}$ and $q^{\prime}$ be the first and last points (respectively) of $p q \cdot T_{k+1}^{\prime}$ in the order from $p$ to $q$. The subarcs $p p^{\prime}$ and $q q^{\prime}$ of $p q$ abut on $T_{k+1}^{\prime}$ from different sides. In $T_{i}+T_{j}+J$ there is an arc $T^{\prime}$ such that $T^{\prime}+T_{k+1}^{\prime}$ is a simple closed curve containing only $p^{\prime}+q^{\prime}$ of $p p^{\prime}+q q^{\prime}$. Hence $T^{\prime}+T_{k+1}^{\prime}$ separates $p$ from $q$. But $(M+Q) \cdot\left(T^{\prime}+T_{k+1}^{\prime}\right)$ separates $p$ from $q$ in $M+Q$ and is a subset of $T_{i}+T_{j}+T_{k+1}$ which is of diameter less than or equal to $3 \epsilon$. Furthermore $\rho\left(T_{i}+T_{j}+T_{k+1}, B\right)<\epsilon$. This involves a contradiction from which the validity of the theorem follows.

The preceding theorem has been formulated so as to parallel as nearly as possible the corresponding theorem for locally connected continua in [1]. A simpler but equivalent statement (suggested by the referee) follows:

Theorem. If the continuum $M$ does not separate $S, Q$ is a component of the interior of $M$, and $p$ belongs to $M-\bar{Q}$, then there is a point $x$ of $\bar{Q}$ which cuts $p$ from $Q$ in $M$.

Corollary. ${ }^{7}$ If the continuum $M$ does not separate $S$, and $p$ and $q$ belong to different components of the interior of $M$, then some point $x$ of $M$ cuts $p$ from $q$ in $M$.

\section{BIBLIOGRAPHY}

1. R. L. Moore, Foundations of point-set theory, Amer. Math. Soc. Colloquium Publications vol. 13, New York, 1932.

2. R. E. Bayse, Simply connected sets, Trans. Amer. Math. Soc. vol. 38 (1935) pp. 341-356.

University of North Carolina and The Institute for Advanced Study

${ }^{6}$ For instance, see Theorem 13 of [2]. There are other results in this paper of relevant interest.

7 Proposed by Professor A. M. Gleason (in conversation). 